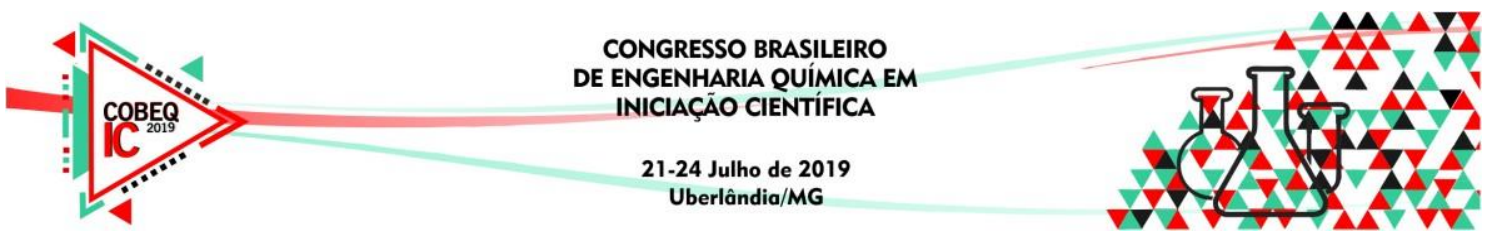

\title{
ANÁLISE EXPERIMENTAL DO TEMPO DE MISTURA NA AGITAÇÃO DE FLUIDOS NEWTONIANOS EM TANQUE EQUIPADO COM IMPULSOR RADIAL TURBINA
}

\author{
B. D. SANTOS ${ }^{1}$, F. PITTA ${ }^{2}$, I. G. GARCIA ${ }^{1}$, J. S. DA SILVA ${ }^{1}$, V. S. ROSA ${ }^{1}$ \\ ${ }^{1}$ Universidade Santa Cecília, Departamento de Engenharia Química \\ ${ }^{2}$ Universidade Santa Cecília, Departamento de Engenharia de Produção \\ E-mail para contato: senhorbreno@outlook.com
}

\begin{abstract}
RESUMO - Nas indústrias químicas e petroquímicas a agitação de fluidos newtonianos em tanques com impulsores mecânicos tem ampla aplicação como armazenadores, trocadores de calor, extratores, reatores químicos, destiladores e flotadores. Os principais parâmetros de projeto são o tempo de mistura e potência consumida, porém a literatura é escassa de informações que predizem as variáveis supracitadas com fluidos newtonianos. $\mathrm{O}$ estudo teve por objetivo analisar experimentalmente o tempo de mistura na agitação de fluidos newtonianos em tanque equipado com impulsores mecânicos. A unidade experimental possui um tanque com volume útil de 10 litros, um motor elétrico com potência de um $1 \mathrm{hp}, 1$ chicana padrão sem orifícios, 1 chicana modificada com 120 orifícios e 1 impulsor radial tipo turbina com 6 pás planas. Os fluidos utilizados foram água, solução de sacarose a $20 \%$ e $50 \%$ e glicerina. Constatou-se que para Reynolds entre 100 e 3000, o uso das chicanas sem orifícios fornecem os menores tempo de mistura e para Reynolds entre 3000 e 100000, as chicanas com orifícios foram mais eficazes.
\end{abstract}

\section{INTRODUÇÃO}

Reatores químicos, trocadores de calor, armazenadores, extratores, flotadores e decantadores são algumas unidades que utilizam tanques com impulsores mecânicos, sendo que o projeto dessas unidades é específico para cada operação supracitada (Rosa et. al., 2017).

No entanto, os parâmetros de tempo de mistura e consumo de potência do impulsor mecânico são comuns em todas as operações envolvendo tanques com agitação e mistura (Moraes Junior e Moraes, 2011).

O tempo de mistura é uma variável importante para projetar o tanque, o qual determina o tempo em que as fases que estão sendo agitadas irão demorar para homogeneizar. Por exemplo, para um tanque sendo empregado para produção de tintas 


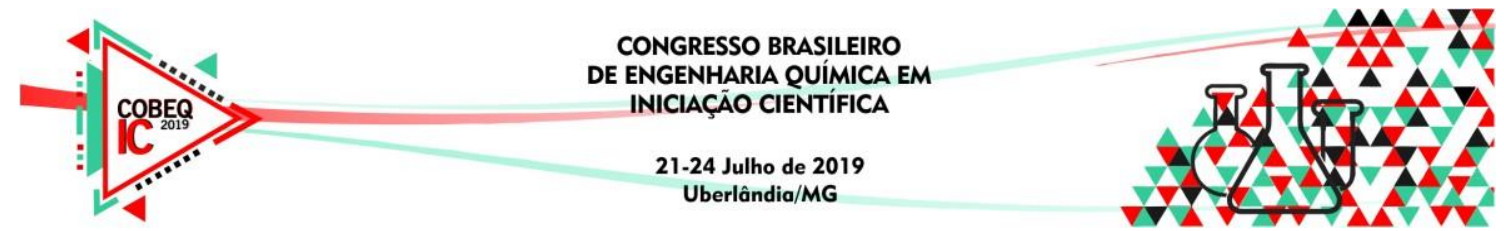

automotivas é interessante que os reagentes contidos nele, se misturem de forma rápida e eficiente, a fim de maximizar a produção do produto (Tatterson, 1991).

O projeto de um tanque baseado no tempo de mistura deve ser executado em associação com o consumo de potência. Baixos tempos de mistura (o que seria ideal), geralmente, necessitam de rotações elevadas do impulsor mecânico, o que leva a um consumo excessivo de potência, o que inviabiliza o custo de um motor elétrico que supra essa especificação (Paul, Atiemo-Obeng e Kresta, 2004).

Na literatura atual, há uma defasagem de equações de previsão do tempo de mistura para condições mais específicas de agitação, como por exemplo, modificações na estrutura dos internos do tanque.

\section{OBJETIVO}

O presente estudou visou analisar o tempo de mistura da agitação de fluidos newtonianos em um tanque com volume de 10 litros equipado com um impulsor radial tipo turbina com 6 pás planas. A unidade também foi dotada de um conjunto de chicanas padrão e um conjunto de chicanas modificadas com orifícios.

\section{METODOLOGIA}

Na Figura 1 está apresentada a unidade experimental utilizada durante a pesquisa, a qual se localiza no Laboratório de Operações Unitárias da Universidade Santa Cecília, em Santos, São Paulo. A unidade experimental foi constituída por um tanque em acrílico com volume útil de 10 litros, um conjunto de chicanas padrão (Figura 2a), um conjunto de chicanas modificada (Figura 2b), um impulsor axial com 4 pás inclinadas a $45^{\circ}$ e um impulsor radial tipo turbina com 6 pás planas.

Utilizou-se também um motor elétrico com potência nominal de $1 \mathrm{hp}$, uma serpentina em espiral para controle de temperatura nos ensaios, a qual possuía acoplada um banho termostático com controle PID e sensores termopares para a captação da temperatura.

Os fluidos newtonianos utilizados foram água, soluções de sacarose a $20 \%$ e 50\%, em massa, e glicerina. Os quais foram submetidos a agitação com rotações entre 100 a 1000 rpm em função de cada impulsor e cada chicana, totalizando assim 120 ensaios, uma vez que se utilizou triplicata.

As dimensões do tanque e dos seus acessórios foram determinadas a partir das recomendações citadas por Rushton, Costich e Everett (Moraes Júnior e Moraes, 2011). Em relação as chicanas modificadas, por ser uma proposta inédita neste trabalho, não foi localizado na literatura nenhuma relação sobre o diâmetro, espaçamento e quantidade de orifícios. Desse modo, foram propostas as seguintes relações: a) diâmetro dos orifícios (Equação 1), b) Espaçamento entre orifícios (Equação 2) e c) Número de orifícios em cada chicana (Equação 3). 


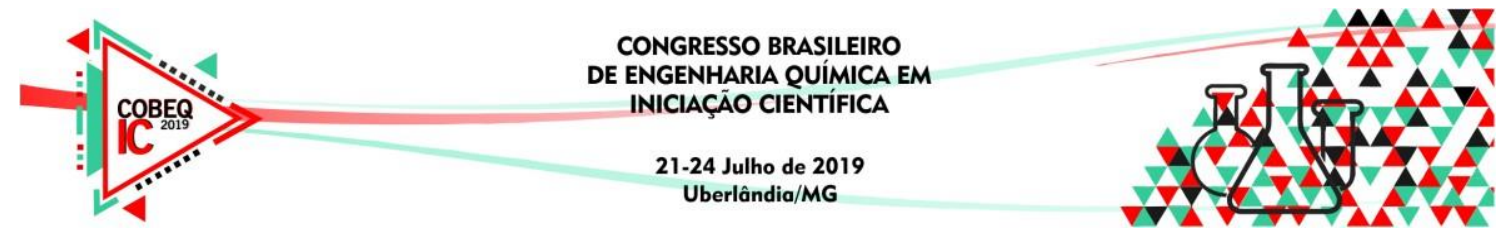

Figura 1 - Unidade Experimental

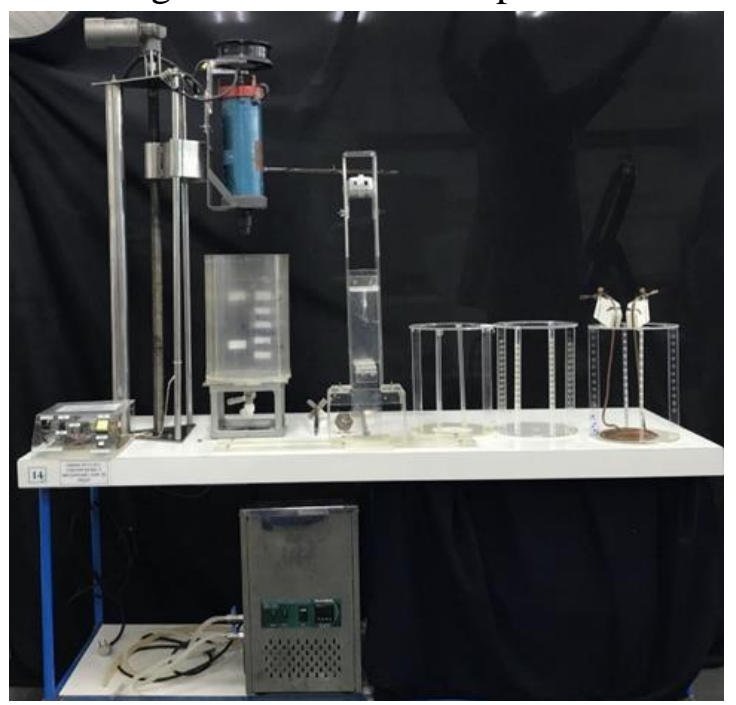

$$
\mathrm{S}_{1}=\frac{\phi}{\mathrm{J}}=\frac{1}{3} \quad \text { (1) } \quad \mathrm{S}_{2}=\frac{\mathrm{N}_{0} \phi}{\mathrm{D}_{\mathrm{t}}}=\frac{1}{2} \quad \text { (2) } \quad \mathrm{S}_{3}=\frac{\mathrm{D}_{0}}{\mathrm{~J}}=\frac{2}{3}
$$

Inicialmente, cada líquido submetido a agitação em uma dada rotação tinha a sua temperatura mantida constante em $20^{\circ} \mathrm{C}$ proveniente de uma serpentina em espiral acoplada a um banho termostático. Simultaneamente, $500 \mathrm{ml}$ do líquido era aquecido até $60^{\circ} \mathrm{C}$ e depois adicionado ao líquido em agitação. Com os termopares, mediu-se a variação de temperatura da mistura em função do tempo até que ocorresse a estabilização da mesma. O tempo em que a temperatura da mistura alcançava o equilíbrio era denominado de tempo de mistura. O procedimento descrito foi repetido para cada líquido em função da rotação e do tipo de chicana.

Figura 2 - (a) Chicana Padrão; (b) Chicana Modificada com 120 orifícios

(a)

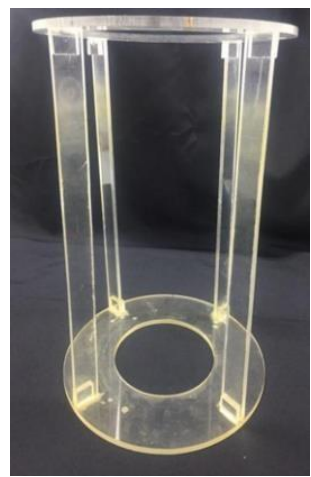

(b)

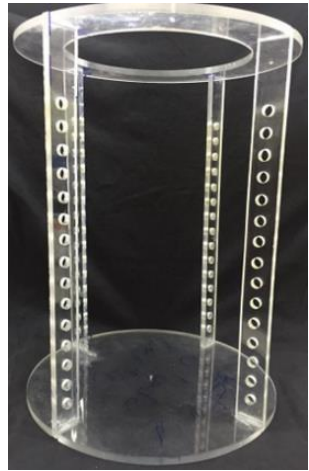




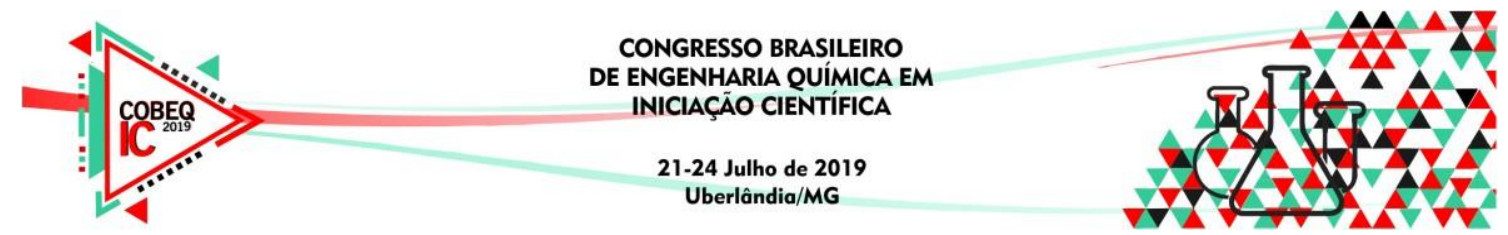

\section{RESULTADOS E DISCUSSÃO}

Na Figura 3 foram plotados os valores do tempo de mistura (os quais foram obtidos conforme o detalhamento na seção metodologia) em função do número de Reynolds para a agitação dos líquidos empregados em relação ao tipo de chicana

Figura 3 - Tempo de mistura em função de Reynolds - chicana sem orifícios e chicana com orifícios

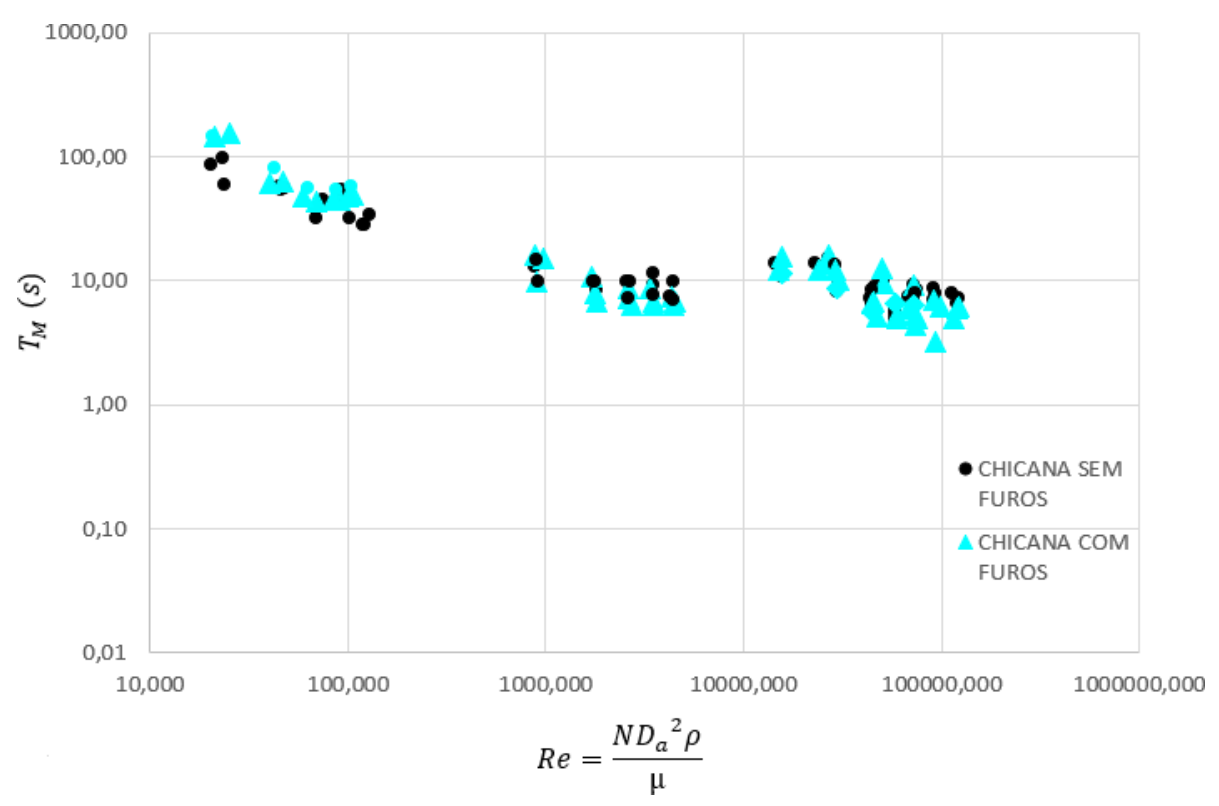

Observou-se que houve uma sobreposição dos resultados com a agitação utilizando a chicana padrão (sem orifícios) e com a chicana modificada (orifícios), o que indica que o tipo de chicana não provoca um efeito significativo na variação do tempo de mistura.

Em termos de projeto, é mais vantajoso utilizar as chicana com orifícios pois a mesma precisa de uma quantidade menor de material para serem confeccionados, o que impacta diretamente no custo do projeto.

Fenomenologicamente, o tempo de mistura é função predominante do número de Reynolds, devido ao escoamento. Desse modo é possível estimar uma expressão para o tempo de mistura, conforme apresentado na Equação 4.

$$
T_{m}=K R e^{a}
$$

A constante K e o expoente a da Equação 4 são obtidas através de regressão não linear e como função do tipo de chicana. Na Tabela 2 estão apresentados os valores obtidos e a análise estatística dos supracitados parâmetros. 


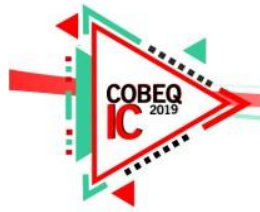

Tabela 2 - Parâmetros da Equação 4 - chicana padrão e chicana modificada

\begin{tabular}{|c|c|c|}
\hline Parâmetros & Chicana padrão & Chicana modificada \\
\hline Constante K & 102,95 & 180,33 \\
\hline Expoente a & $-0,24$ & $-0,31$ \\
\hline $\mathrm{R}^{2}$ & 0,78 & 0,78 \\
\hline Estatística t & 31,54 & 27,53 \\
\hline Valor de P & $3,30.10^{-38}$ & $5,44.10^{-35}$ \\
\hline
\end{tabular}

A partir dos parâmetros presentes na Tabela 2, verificou-se que ambos os modelos possuem um coeficiente de determinação $\left(\mathrm{R}^{2}\right)$ de 0,78 , o que é satisfatório. Nos dois casos, a estatística t foi maior que o valor de $\mathrm{P}$, o que indica que os parâmetros das equações são significativos a nível populacional.

Na Figura 4 foram plotados os modelos obtidos em função do número de Reynolds. Pode-se verificar que para Reynolds entre 100 e 3000, os menores valores do tempo de mistura foram obtidos com a chicana sem orifícios. No entanto, para Reynolds no intervalo de 3000 a 100000 (turbulência), a chicana com orifícios se mostrou mais eficiente, oferecendo os menores tempo de mistura.

Figura 4 - Comparação dos modelos

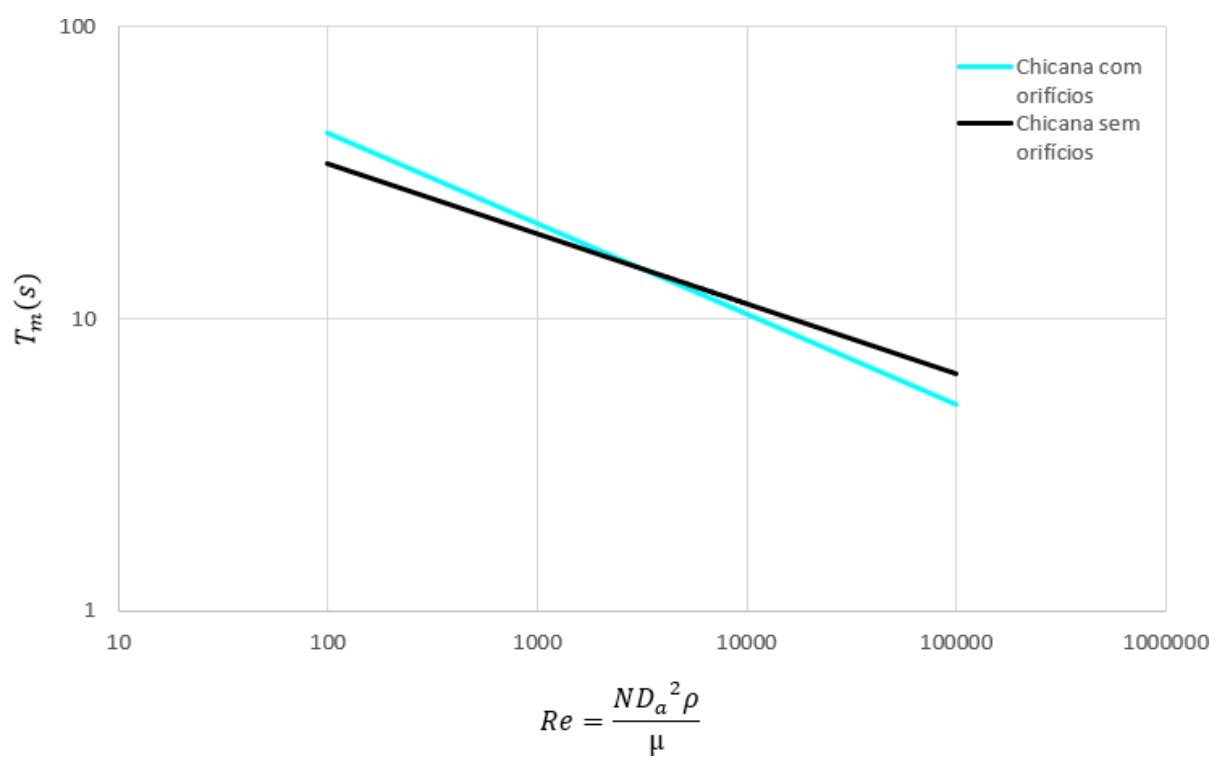

\section{CONCLUSÕES}

Pode-se concluir que:

a) Recomenda-se o uso da chicana com orifícios para operações em regime turbulento (Reynolds acima de 3000) com o uso do impulsor radial tipo turbina; 
b) Os modelos obtidos para a predição do tempo de mistura são válidos para agitação de líquidos newtonianos, com o uso de impulsor radial e Reynolds no intervalo de 100 a 100000 ;

c) Em termos de projeto, as operações de agitação ocorrem na turbulência, desse modo, o uso das chicanas com orifícios irá fornecer um menor custo-benefício na sua confecção devido a sua menor massa quando comparada com a chicana padrão.

\section{NOMENCLATURA}

\begin{tabular}{|c|c|c|c|}
\hline $\mathrm{a}$ & Expoente da Equação 4 & $\mathrm{~N}_{\mathrm{o}}$ & Número de orifícios \\
\hline $\mathrm{D}_{\mathrm{a}}$ & Diâmetro do impulsor $(\mathrm{m})$ & $\mathrm{T}_{\mathrm{m}}$ & Tempo de mistura $(\mathrm{s})$ \\
\hline $\mathrm{D}_{\mathrm{o}}$ & Diâmetro do orifício $(\mathrm{m})$ & $\mathrm{Re}$ & Reynolds \\
\hline $\mathrm{D}_{\mathrm{t}}$ & Diâmetro do tanqye $(\mathrm{m})$ & $\Phi$ & $\begin{array}{c}\text { Espzçamento entre os } \\
\text { orifícios }(\mathrm{m})\end{array}$ \\
\hline $\mathrm{J}$ & Largura da chicana $(\mathrm{m})$ & $\rho$ & Massa específica $\left(\mathrm{kg} / \mathrm{m}^{3}\right)$ \\
\hline $\mathrm{K}$ & Constante da Equação 4 & $\mu$ & Viscosidade $(\mathrm{Pa} . \mathrm{s})$ \\
\hline $\mathrm{N}$ & Rotação (rpm) & & \\
\hline
\end{tabular}

\section{REFERÊNCIAS}

ROSA, V.S., TAQUEDA, M. E. S., PAIVA, J. L., MORAES, M. S., MORAES JÚNIOR, D. "Nusselt's correlations in agitated tanks using the spiral coil with Rushton turbine and PBT $45^{\circ}$ impeller. Comparison with tanks containing vertical tube baffles." Applied Thermal Engineering, 110, p. 1331-1342, 2017;

MORAES JUNIOR, D., MORAES, M.S., "Laboratório de Operações Unitárias I", $1^{\circ}$ Ed., Brasil, Edição do Autor, 2011;

PAUL, E. L., e ATIEMO-UBENG, S. M., "Handbook of mixing", 1ª Ed., WileyInterscience, 2004;

TATTERSON, G.B., "Fluid Mixing and Gas Dispersion in Agitated Tanks", McGraw-Hill Book Co, 1991. 